\title{
CHEMICAL COMPOSITION AND CIRCUMSTELLAR SHELLS OF CARBON STARS - ANY OBVIOUS RELATIONS ?
}

\author{
Kjell Eriksson ${ }^{1}$, Bengt Gustafsson ${ }^{1,2}$ and Hans Olofsson ${ }^{3}$ \\ 1 Uppsala Astronomical Observatory, Box 515, \\ S-751 20 Uppsala, Sweden \\ 2 Stockholm Observatory, S-133 00 Saltsjöbaden, Sweden \\ 3 Onsala Space Observatory, S-439 00 Onsala, Sweden
}

Evidence for circumstellar absorption around the warm N-type carbon star TX Piscium was found in a high-resolution IUE spectrum by Eriksson et al. (1986). This investigation also included the search, with a positive result, for $\mathrm{CO} \mathrm{J}=1-0$ emission from the circumstellar shell. From the $\mathrm{Mn} I$ absorption and $\mathrm{CO}$ emission a column density of about $10^{20}-10^{22}$ $\mathrm{H}$ atoms per $\mathrm{cm}^{2}$ was estimated, as well as a mass loss rate around $10^{-7}$ $10^{-6} \mathrm{M}$ per year.

Lambe̊rt et al. (1986) have recently determined CNO abundances and ${ }^{12} \mathrm{C} /{ }^{13} \mathrm{C}$ ratios for 30 bright, galactic $\mathrm{N}$-type stars. From this sample we have selected twelve stars with different chemical profiles to survey

TABLE I Stellar parameters from Lambert et a1. (1986)

\begin{tabular}{|c|c|c|c|c|c|}
\hline Star & $\begin{array}{r}\mathrm{T}_{\text {eff }} \\
(\mathrm{K})\end{array}$ & ${ }^{12} \mathrm{C} /{ }^{13} \mathrm{C}$ & $\lg \frac{C-0}{0}$ & {$[\mathrm{~N} / \mathrm{H}]$} & {$[0 / \mathrm{H}]$} \\
\hline Z Psc & 2870 & 55 & -1.85 & -.39 & -.23 \\
\hline U Cam & 2530 & 97 & -.52 & -.42 & -.42 \\
\hline Y Tau & 2600 & 58 & -1.40 & -.17 & -.19 \\
\hline BL Ori & 2960 & 57 & -1.41 & +.05 & -.29 \\
\hline UU Aur & 2825 & 52 & -1.20 & +.15 & -.18 \\
\hline VY UMa & 2855 & 44 & -1.22 & -.31 & -.29 \\
\hline Y CVn & 2730 & 3.5 & -1.06 & -.12 & -.40 \\
\hline RY Dra & 2500 & 3.6 & -.74 & -.05 & -.38 \\
\hline $\mathrm{T}$ Lyr & 2380 & 3.2 & -.54 & -.83 & -.50 \\
\hline UX Dra & 2900 & 32 & -1.34 & -.12 & -.21 \\
\hline 460 Cyg & 2845 & 61 & -1.21 & -.06 & -.32 \\
\hline TX Psc & 3030 & 43 & -1.57 & -.27 & -.10 \\
\hline $\mathrm{Z}$ Cas & 285 & & 0 & +.01 & +.07 \\
\hline
\end{tabular}

Results from CO J=1-0 observations at Onsala Space Observatory

\begin{tabular}{|c|c|c|c|}
\hline $\begin{array}{l}\mathrm{T}_{\mathrm{mb}} \\
(\mathrm{K})\end{array}$ & $\begin{array}{c}\mathrm{v}_{\mathrm{LSR}} \\
(\mathrm{km} / \mathrm{s})\end{array}$ & $\begin{array}{c}v_{\exp } \\
(\mathrm{km} / \mathrm{s})\end{array}$ & Notes \\
\hline 0.20 & 12.8 & 4.2 & \\
\hline$\sim 0.2$ & $\sim 10$ & $\sim 18$ & 1,2 \\
\hline $\begin{array}{c}\sim 0.25 \\
?\end{array}$ & $\sim 15$ & $\sim 13$ & $\begin{array}{l}1,3 \\
1\end{array}$ \\
\hline $\begin{aligned} & 0.46 \\
\leqq & 0.2\end{aligned}$ & 6.7 & 12.4 & 4 \\
\hline $\begin{array}{r}0.36 \\
0.15\end{array}$ & $\begin{array}{r}19.7 \\
-\quad 4.9\end{array}$ & $\begin{array}{r}9.1 \\
10.9\end{array}$ & 5 \\
\hline$\leqq 0.08$ & & & \\
\hline $\begin{array}{l}0.19 \\
0.27 \\
0.25\end{array}$ & $\begin{array}{l}13.7 \\
26.3 \\
13.1\end{array}$ & $\begin{array}{r}7.1 \\
13.1 \\
12.5\end{array}$ & \\
\hline$\leqq 0.08$ & & & \\
\hline
\end{tabular}

Notes:

1. Interstellar lines

2. Previously detected: ZDC: $0.16 / 8.5 / 22.0 \quad \mathrm{~J}=1-0$

3. Previously detected: ZDC: $0.17 / 15.9 / 10.1 \quad \mathrm{~J}=1-0$

4. Previously detected: ZD: $\quad 0.66 / 3.6 / 11.5 \mathrm{~J}=2-1$ ZDC: $0.26 / 7.6 / 12.4 \mathrm{~J}=2-1$ K: $\quad 0.06 / 7.0 / 13.4 \mathrm{~J}=1-0$
5. Previously detected:

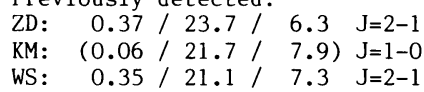

In 2-5 the numbers given are $\mathrm{T}_{\mathrm{mb}} / \mathrm{v}_{\mathrm{LSR}} / \mathrm{v}_{\exp }$

K Knapp(1986): Princeton Obs. preprint 167

KM Knapp \& Morris(1985): Ap.J. 292, 640

WS Wannier \& Sahai(1985): JPL preprint 106

ZD Zuckerman \& Dyck(1986): Ap.J. 304, 394

ZDC Zuckerman,Dyck \& C1aussen(1986):ApJ 304,401 
their possible $C O$ emission in the $J=1-0$ transition with the Onsala $20 \mathrm{~m}$ telescope. The observations were performed in December 1985 and April 1986. We detected CO emission from eight of the stars; the results are presented in Table $I$ and two examples are displayed in Figure 1. The main beam brightness temperature, $T_{m b}$, is the antenna temperature divided by the main beam efficiency ${ }^{m b}(\simeq 0.3)$, and antenna and radome transmission factors. Four of the stars have been detected in $\mathrm{CO}$ by other groups independently.

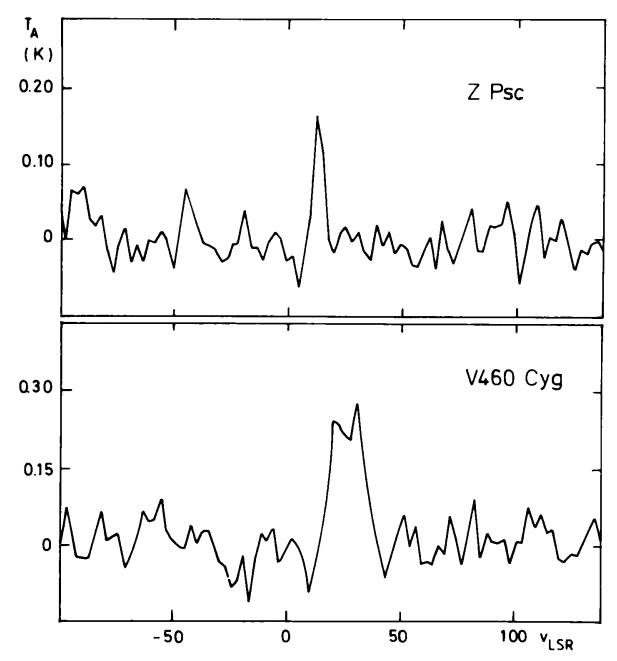

Figure 1: CO J=1-0 emission profiles for two N-type stars in our sample.

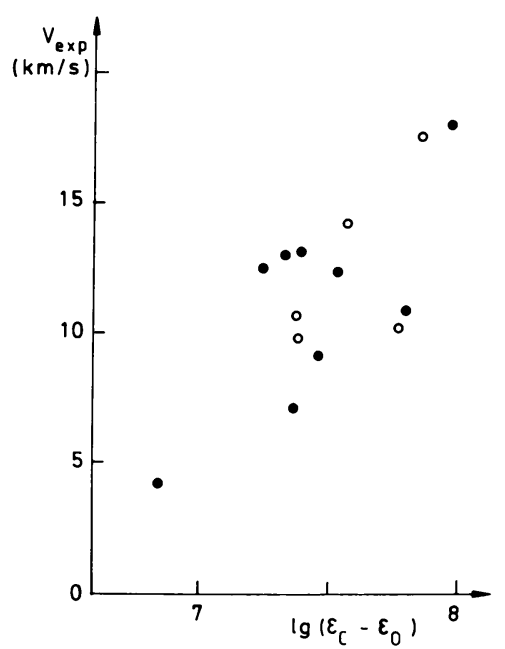

Figure 2: CO expansion velocity vs. carbon excess. Abscissa normalized such that $l g \varepsilon_{H}=12$. Open symbols are from other investigations.

We have investigated whether the shell emission and expansion velocities correlate with the chemical parameters, the effective temperatures or the flux excess at $11 \mu \mathrm{m}$ as measured on IRAS low resolution spectra. No significant correlations were found. However, there may be a tentative correlation between the expansion velocity and the carbon excess ( $r$ elative to oxygen), Figure 2. This correlation, which has the right direction if radiation forces on dust grains is an important mass loss mechanism, is worth further study.

\section{References:}

Eriksson,K., Gustafsson,B., Johnson,H.R., Querci,F., Querci,M.,

Baumert,J.H., Carlsson,M. \& Olofsson,H. 1986: Astron. Astrophys.161,305 Lambert,D.L., Gustafsson,B., Eriksson,K. \& Hinkle,K.H. 1986: Astrophys. J. Suppl., September issue 